\title{
ALGAS MARINHAS BENTÔNICAS DO MUNICÍPIO DE ILHÉUS, BAHIA, BRASIL
}

\author{
José Marcos de Castro NUNES, Ana Cristina Caribé dos SANTOS, \\ Augusto MINERVINO y Keila Santana BRITO
}

RESUMO. Algas marinhas bentônicas do município de Ilhéus, Bahia, Brasil. Levantamento qualitativo das algas marinhas bentônicas, totalizando 77 táxons distribuídos em 22 Chlorophyta, 15 Phaeophyta e 40 Rhodophyta. Pterosiphonia pennata (C. Agardh) Falkenberg e Caulerpa taxifolia (H. West in Vahl) C. Agardh constituem-se em novas citações para o Estado da Bahia.

Palavras chave. Algas marinhas bentônicas, Ilhéus, Bahia, Brasil.

ABSTRACT. Marine benthic algae of Ilhéus county, Bahia State, Brazil. In this qualitative survey of benthic algae, were identified 77 taxa distributed into 22 Chlorophyta, 15 Phaeophyta and 40 Rhodophyta. Pterosiphonia pennata (C. Agardh) Falkenberg and Caulerpa taxifolia (H. West in Vahl) C. Agardh were recorded in the first time for Bahia State.

Key words. Seaweeds, Ilhéus, Bahia, Brazil.

\section{INTRODUÇÃO}

O primeiro levantamento sistemático de algas marinhas do litoral baiano foi realizado e publicado Martins et al. (1991) fazendo referência a 33 táxons de clorofíceas marinhas do município de Salvador. Recentemente, foram publicados outros levantamentos da ficoclora marinha bentônica baiana, a exemplo de Nunes (1997), que estudou quatro famílias de rodofíceas das praias de Placafor e Itapoã em Salvador, Altamirano \& Nunes (1997) apresentaram levantamento das algas da praia de Itacimirim no município de Camaçari e Nunes (1998a), que tratou das rodofíceas do mesmo município. Nunes (1998b) compilou todos os trabalhos realizados no Estado, acrescentando 35 novas citações para o litoral baiano, sendo algumas, novas referências para o litoral nordestino.

Especificamente para o litoral sul e extremo sul do estado da Bahia, são citados os trabalhos de Taylor (1930 e 1931), Joly et al. (1967, 1969a e 1969b), Yamaguishi-Tomita (1970), Oliveira Filho et al. (1979), Guimarães et al. (1981) realizados no município de Caravelas, principalmente no Arquipélago de Abrolhos. Posteriormente, Teixeira et al. (1985) estudaram as algas de profundidade da costa sudeste do Brasil onde incluiu material coletado no sul da Bahia. Ugadim $(1987,1993)$ realizou estudos taxonômicos sobre os gêneros 
Gelidium Lamouroux e Pterocladia J. Agardh, incluindo material coletado nos municípios de Ilhéus, Santa Cruz de Cabrália, Porto Seguro, Prado e Alcobaça. Corallina panizzoi Schnetter et Richter foi referida pela primeira vez para o Brasil por Buys \& Széchy (1996), incluindo material coletado no município de Ilhéus em 1965. Material coletado nos municípios de Ilhéus e Uruçuca foi referido por Nunes (1999) ao estudar as algas pardas da Região Metropolitana de Salvador. Contudo, estes trabalhos restringem-se a listas, adições à flora brasileira ou estudos com táxons específicos que foram coletados na Região.

O presente trabalho tem como objetivo contribuir para o conhecimento da biodiversidade das macroalgas marinhas do Estado da Bahia, fornecendo subsídios para o aproveitamento racional e preservação dos estoques naturais, bem como o mapeamento das áreas de distribuição das espécies ao longo do litoral brasileiro, e integra o Projeto "Levantamento das Algas Marinhas Bentônicas da Mesorregião Sul Baiana", desenvolvido no Departamento de Ciências Biológicas da Universidade Estadual de Santa Cruz em parceria com a Universidade Federal da Bahia, Universidade do Estado da Bahia, e em colaboração com o Instituto de Botânica do Estado de São Paulo.

\section{MATERIAL E MÉTODOS}

O município de Ilhéus (14²7's x $39^{\circ} 03^{\prime} \mathrm{W}$ ) está localizado na região sul do estado da Bahia e com aproximadamente $70 \mathrm{~km}$ de litoral. Sua geomorfologia costeira se apresenta sob a forma quase que exclusivamente de praias arenosas com escassos e insipientes substratos rochosos na região intermareal.

O litoral sul da Bahia é uma área de desembocadura de rios de grande calado destacando-se o rio Jequitinhonha, Pardo e Contas, sendo que, no perímetro urbano da cidade de Ilhéus, desembocam três outros rios de menores proporções, o rio Almada, Cachoeira e Santana. Esse regime fluvial confere a água oceânica altos índices de turbidez devido a grande carga de matéria orgânica introduzida por estes rios através do aumento do volume de sedimentos terrígenos.

O material foi coletado nas praias do Aeroporto, Olivença e Morro de Pernambuco, no Município de Ilhéus (fig. 1), no período do abril de 1994 a junho de 1996, nos períodos estacionais (inverno, outono, primavera e verão). Em duas dessas praias, Aeroporto e Olivença foram realizadas quatro coletas, e na praia do Morro de Pernambuco, três.

As coletas foram feitas nos dias de marés mais baixas de sizígia, no mesolitoral, sendo este, subdividido em pontos de coleta de acordo com o hidrodinamismo, ou seja, maior ou menor incidência de ondas: modo batido, modo moderadamente agitado ou poças de maré.

Os exemplares foram coletados com espátulas e acondicionados em sacos plásticos devidamente etiquetados e preservados de acordo com Cordeiro-Marino et al. (1984). O material estudado encontra-se depositado e registrado no Herbário Alexandre Leal Costa (ALCB) do Instituto de Biologia da Universidade Federal da Bahia.

O agrupamento dos táxons em famílias e ordens seguiu-se Wynne (1998).

Para cada espécie são fornecidos os seguintes dados:

a) Distribuição: AE, Praia do Aeroporto; MP, Praia do Morro de Pernambuco e OL, Praia de Olivença.

b) Reprodução: OI, Órgãos intercalares; OP, Órgãos pluriloculares; ESP, Esporângios; Z, Zoósporos; FEM, Feminina; MASC, Masculina; MONO, Monóica; T, Tetrasporângios e C, Cistocarpo.

c) Ecologia: E, Epífita; RO, Substrato rochoso; ZB, Zona batida; MA, Moderadamente agitado e PÇ, poça.

d) Número de Herbário. 


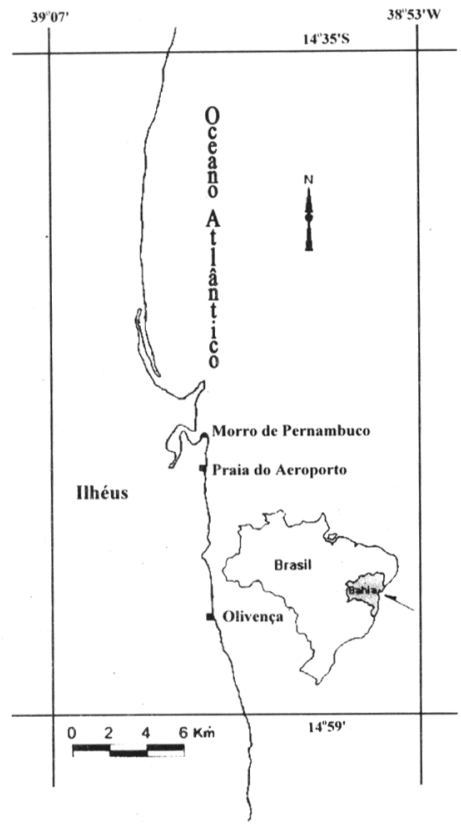

Figura 1. Mapa com a localizaçao das praias inventariadas.

\section{RESULTADOS E DISCUSSÃO}

Nas praias amostradas foram coletados e identificados 77 táxons infragenéricos: sendo 40 Rhodophyta distribuídos em 10 ordens e 15 famílias, das quais Rhodomelaceae, Ceramiaceae e Corallinaceae foram melhor representadas com 11,5 e 5 táxons respectivamente; 15 Phaeophyta com 4 ordens e 4 famílias, sendo Dictyotaceae e Sargassaceae as mais representativas, ambas com 5 táxons; 22 Chlorophyta com 3 ordens e 9 famílias, das quais Caulerpaceae, é a melhor representada com 6 táxons.

Das praias amostradas, a praia do Aeroporto apresentou maior ocorrência de número de táxons (64), seguido de Olivença (60) e Morro de Pernambuco (51).

Foram observadas algumas diferenças na ocorrência dos táxons nos diversos pontos de coleta de acordo com o hidrodinamismo: em local de modo batido no mesolitoral foram assinalados 62 táxons, moderadamente agitado 44 e poças 40 táxons. Apenas 20 táxons foram encontrados em todos os pontos de amostragem.

A maioria dos táxons estudados apresentaram exemplares férteis. Em Chlorophya, apenas Ulva fasciata apresentou zoósporos biflagelados em exemplares coletados. A ausência de plantas férteis na maioria dos exemplares de clorofíceas também foi verificado por Kanagawa (1984) e Martins et al. (1991).

Apenas 11 espécies ocorrem exclusivamente como epífitas.

Pterosiphonia pennata e Caulerpa taxifolia foram referidas pela primeira vez para o estado da Bahia.

\section{LISTA DE TÁXONS}

\section{CHLOROPHYTA}

\section{ULVALES}

Ulvaceae

Enteromorphaflexuosa (Wulfen ex Roth) J. Agardh AE, OL; RO, ZB, MA. (ALCB 34630).

Ulva fasciata Delile

AE, MP, OL; Z; RO, ZB, MA, PÇ. (ALCB 22329).

\section{CLADOPHORALES}

Anadyomeniaceae

Anadyomene stellata (Wulfen) C. Agardh

AE, MP, OL; RO, ZB, MA, PÇ. (ALCB 34631).

\section{Cladophoraceae}

Chaetomorpha antennina (Bory) Kützing

AE, MP, OL; RO, ZB, MA, PÇ. (ALCB 22288).

Cladophora prolifera (Roth) Kützing

AE, MP, OL; E, ZB, PÇ. (ALCB 34632). 
C. vagabunda (Linnaeus) van den Hoek

AE; RO, MA. (ALCB 34633).

Siphonocladaceae

Cladophoropsis membranacea (C. Agardh) B $\varnothing$ rgesen 34634).

AE, MP, OL; E, RO, ZB, MA, PÇ. (ALCB

Valoniaceae

Dictyosphaeria versluysii Weber-Van Bosse

AE, OL; RO, MA. (ALCB 34635).

Valonia aegagropila $\mathrm{C}$. Agardh

OL; RO, ZB, MA. (ALCB 34636).

\section{BRYOPSIDALES}

Bryopsidaceae

Bryopsis corymbosa J. Agardh

AE, OL; RO, PÇ. (ALCB 34637).

B. pennata Lamouroux

AE, OL; RO, PÇ. (ALCB 34638).

Codiaceae

Codium intertextum Collins \& Hervey

AE, MP, OL; RO, ZB. (ALCB 34639).

C. isthmocladum Vickers

AE, MP, OL; RO, ZB, MA, PÇ. (ALCB 22287).

C. taylorii Silva

AE; RO, ZB, PÇ. (ALCB 34640).

Caulerpaceae

Caulerpa cupressoides (West in Vahl) C. Agardh AE, MP, OL; RO, ZB, PÇ. (ALCB 22303).

C. fastigiata Montagne

AE; RO, PÇ. (ALCB 34641).

C. prolifera (Forsskål) Lamouroux

AE, MP, OL; RO, ZB, PÇ. (ALCB 22319).
C. racemosa (Forsskål) J. Agardh

OL; RO, ZB, MA. (ALCB 34643).

C. sertularioides (S. G. Gmelin) Howe

MP, OL; RO, ZB, MA, PÇ. (ALCB 34644).

C. taxifolia (H. West in Vahl) C. Agardh

AE, MP, OL; RO, ZB, PÇ. (ALCB 34642).

Udoteaceae

Boodleopsis pusilla (Collins) W. Taylor, Joly \& Bernatowicz

AE; E, RO, ZB. (ALCB 34645).

Halimeda discoidea Decaisne

AE, MP, OL; RO, ZB, MA, PÇ. (ALCB 22320).

\section{PHAEOPHYTA}

\section{ECTOCARPALES}

Ectocarpaceae

Bachelotia antillarum (Grunow) Gerloff AE, MP, OL; OI; RO, MA, PÇ. (ALCB 31543).

Feldmannia irregulares (Kützing) Hamel AE; OP; E, RO, AB. (ALCB 34646).

Hincksia breviarticulata (J. Agardh) P. C. Silva AE; OP; E, PÇ. (ALCB 34647).

H. mitchelliae (Harvey) Hamel

AE; OP; E, ZB, PÇ. (ALCB 34648).

\section{SCYTOSIPHONALES}

\section{Scytosiphonaceae}

Chnoospora minima (Hering) Papenfuss

AE, MP, OL; OP; RO, ZB, MA. (ALCB 34649).

\section{DICTYOTALES}

Dictyotaceae

Dictyopteris delicatula Lamouroux

AE, MP, OL; ESP; E, ZB, MA, PÇ. (ALCB 34650). 
Dictyota menstrualis (Hoyt) Schnnetter, Hornig \& Weber-Peukert MP; ESP, FEM; RO, MA. (ALCB 34651).

Padina boergesenii Allender \& Kraft AE; ESP, FEM, MASC; RO, ZB, PÇ. (ALCB 31503).

P. gymnospora (Kützing) Sonder

AE, MP, OL; ESP, FEM; RO, ZB, MA, PÇ. (ALCB 22488).

Spatoglossum schroederi (C. Agardh) Kützing AE, OL; ESP, MASC; RO, MA. (ALCB 34652)

\section{FUCALES}

Sargassaceae

Sargassum cymosum $\mathrm{C}$. Agardh

MP, OL; FEM, MASC; RO, ZB, MA, PÇ. (ALCB 34627).

S. cymosum C. Agardh var. nanum E. de Paula \& E.

C. Oliveira

MP, OL; FEM, MASC, RO, ZB. (ALCB 34619).

S. filipendula $\mathrm{C}$. Agardh 34614).

OL; FEM, MASC; RO, ZB, MA, PÇ. (ALCB

S. rigidulum Kützing

AE, MP, OL; MONO; RO, ZB, MA. (ALCB 34616).

S. vulgare C. Agardh

AE, MP, OL; MONO; RO, ZB, MA, PÇ. (ALCB 34653).

\section{RHODOPHYTA}

\section{PORPHYRIDIALES}

Porphyridiaceae

Stylonema alsidii (Zanardini) K. M. Drew AE, MP; E, ZB, MA. (ALCB 34654).

\section{ERYTHROPELTIDALES}

Erythrotrichiaceae

Erythrotrichia carnea (Dillwyn) J. Agardh AE, MP, OL; E, ZB, MA. (ALCB 34655).

\section{CORALLINALES}

Corallinaceae

Amphiroa beauvoisii Lamouroux AE, MP,OL; T; RO, ZB. (ALCB 22331).

A. fragilissima (Linnaeus) Lamouroux AE, MP, OL; T; RO, ZB, MB. (ALCB 34656).

Corallina panizzoi Schnetter \& Richter

AE, MP, OL; T; RO, ZB, MA. (ALCB 22292).

Haliptilon subulatum (Ellis \& Solander) Johansen AE, MP, OL; T, C; RO, ZB, MA, PÇ. (ALCB 22282).

Jania adhaerens Lamouroux

AE, MP, OL; T; RO, ZB, MA. (ALCB 34657).

\section{GELIDIALES}

\section{Gelidiaceae}

Gelidium pusillum (Stackhouse) Le Jolis

AE, MP, OL; T; RO, ZB, MA, PÇ. (ALCB 34658).

\section{Gelidiellaceae}

Gelidiella acerosa (Forsskål) J. Feldmann \& Hamel $\mathrm{AE}, \mathrm{MP}, \mathrm{OL} ; \mathrm{T} ; \mathrm{RO}, \mathrm{ZB}, \mathrm{MA}, \mathrm{PÇ}$. (ALCB 22291).

\section{NEMALIALES}

Galaxauraceae

Galaxaura marginata (Ellis \& Solander) Lamouroux AE, MP, OL; RO, ZB, MA, PÇ. (ALCB 22328). 


\section{GIGARTINALES}

Gigartinaceae

Chondracanthus acicularis (Roth) Fredericq MP, OL; RO, ZB. (ALCB 22330).

Hypneaceae

Hypnea cervicornis J. Agardh

AE, MP, OL; T, C; RO, ZB. (ALCB 34659).

H. musciformis (Wulfen in Jacquin) Lamouroux

AE, OL; T, C; E, ZB, MA, PÇ. (ALCB 22285).

Rhizophyllidaceae

Ochtodes secundiramea (Montagne) Howe

AE; C; RO, MA. (ALCB 34660).

\section{HALYMENIALES}

Halymeniaceae

Cryptonemia luxurians (C. Agardh) J. Agardh AE, MP, OL; RO, ZB, MA, PÇ. (ALCB 22286).

Grateloupia filicina (Lamouroux) C. Agardh AE, MP; RO, ZB. (ALCB 34661).

\section{GRACILARIALES}

Gracilariaceae

Gracilaria caudata J. Agardh

AE, MP, OL; T, C; RO, ZB, PÇ. (ALCB 22283).

G. cervicornis (Turner) J. Agardh 22289).

$\mathrm{AE}, \mathrm{MP}, \mathrm{OL} ; \mathrm{T} ; \mathrm{RO}, \mathrm{ZB}, \mathrm{MA}, \mathrm{PC}$. (ALCB

G. domingensis (Kützing) Sonder ex Dickie

AE, MP, OL; T, C, MASC; RO, ZB, MA. (ALCB 22334).

Hydropuntia cornea (J. Agardh) M. J. Wynne AE, OL; T, C; RO, ZB, MA. (ALCB 22293).

Gelidiopsis variabilis (Greville ex Agardh) F. Schmitz
MP, OL; RO, ZB. (ALCB 34662).

\section{RHODYMENIALES}

Rhodymeniaceae

Botryocladia occidentalis (Børgesen) Kylin AE, OL; RO, ZB, MA, PÇ. (ALCB 22310).

\section{CERAMIALES}

\section{Ceramiaceae}

Aglaothamnion felipponei (Howe) Aponte OL; T; E, ZB. (ALCB 34663).

Centroceras clavulatum (C. Agardh in Kunth) Montagne in Durieu de Maisonneuve AE, MP, OL; T; E, ZB, MA, PÇ. (ALCB 22309).

Centrocerocolax ubatubensis Joly AE; E, ZB. (ALCB 34664).

Spyridia filamentosa (Wulfen) Harvey in Hooker MP, OL; T; RO, MA. (ALCB 34665).

S. hypnoides (Bory in Belanger) Papenfuss AE, MP; T; E, RO, ZB, MA. (ALCB 34666).

\section{Dasyaceae}

Heterosiphonia gibbesii ( Harvey) Falkenberg MP, OL; T; E, RO, ZB, MA. (ALCB 22323).

\section{Rhodomelaceae}

Acanthophora spicifera (Vahl) Børgesen AE, MP; T, MASC; RO, ZB, PÇ. (ALCB 34667).

Bostrychia tenella (Vahl) J. Agardh AE; T; RO, PÇ. (ALCB 34668).

Bryocladia cuspidata (J. Agardh) De Toni AE, MP, OL; T; RO, ZB. (ALCB 34669).

Bryothamnion seaforthii (Turner) Kützing AE, MP, OL; T, C; RO, ZB. (ALCB 22295).

B. triquetrum (S. G. Gmelin) Howe 
AE, MP, OL; T; RO, ZB. (ALCB 34670).

Digenia simplex (Wulfen) C. Agardh

AE, MP, OL; T; RO, ZB, PÇ. (ALCB 22308).

Herposiphonia secunda (C. Agardh) Falkenberg

AE; T, C; E, PÇ. (ALCB 34671)

Laurencia flagellifera $\mathrm{J}$. Agardh

AE, MP, OL; T; RO, ZB. (ALCB 22299).

L. papillosa (C. Agardh) Greville

AE, MP, OL; T; RO, ZB, MA. (ALCB 22301).

Osmundaria obtusiloba (C. Agardh) R. E. Norris AE, MP, OL; T; RO, ZB, PÇ. (ALCB 22300).

Pterosiphonia pennata (C. Agardh) Falkenberg AE; RO, ZB. (ALCB 34672).

AGRADECIMENTOS. À Dra. Mutue Toyota Fujii da Seção de Ficologia do Instituto de Botânica de São Paulo, pela orientação de Estagiários e confirmação de táxons, ao Programa Institucional de Bolsas de Iniciação Científica - PIBIC-CNPq/ UESC pela concessão de Bolsas.

\section{REFERÊNCIAS BIBLIOGRÁFICAS}

ALTAMIRANO, M. y J. M. de C. NUNES -1997Contribuciones al macrofitobentos del municipio de Camaçari (Bahia, Brasil). Acta Bot. Malacitana 22: 211-215.

BUYS, S. C. \& M. T. M. de SZÉCHY. 1996. Corallina panizzoi Schnetter et Richter (Corallinales, Rhodophyta) en el litoral brasilero. Revista Brasileira de Biologia 57(1): 71-77.

CORDEIRO-MARINO, M., N. YAMAGUISHITOMITA \& S. M. P. B. GUIMARÃES -1984Algas. 1.3. Algas marinhas bentônicas. In Técnicas de coleta, preservação e herborização de material botânico (Fidalgo, O. \& Bononi, V. L. R. coord.). São Paulo, Instituto de Botânica. Manual no. 4. p. 1-62

GUIMARÃES, S. M. P. B., M. CORDEIRO-MARINO $\&$ N. YAMAGUISHI-TOMITA -1981- Deep water Phaeophyceae and their epiphytes from northeastern and southeastern Brazil. Revista brasileira de Botânica 4: 95-113.

JOLY, A. B., E. C. de OLIVEIRA FILHO \& W. NARCHI -1969a- Projeto de criação de um Parque Nacional Marinho na Região de Abrolhos, Bahia. Anais da Academia brasileira de Ciências (supl.), 41: 247-251.

JOLY, A. B., Y. UGADIM, J. J. G. DIAZ., N. YAMAGUISHI-TOMITA, M. CORDEIROMARINO \& Y. YONESHIGUE-BRAGA 1969b-Additions to the marine flora of Brazil X. Rickia 4: 61-74.

JOLY, A. B., Y. UGADIM, E. C. de OLIVEIRAFILHO \& M. CORDEIRO-MARINO -1967Additions to the marine flora of Brazil VI. Boletim da Faculdade de Filosofia Ciências e Letras da Universidade de São Paulo 305 (bot.) (2): 171-194.

KANAGAWA, A. I -1984-Cloroficeas marinhas bentônicas do Estado da Paraíba, Brasil. Tese de Doutorado, Universidade de São Paulo. 470p.

MARTINS, D. V., M. CORDEIRO-MARINO, N. B. BOCCANERA \& J. M. de C. NUNES -1991Clorofíceas marinhas bentônicas do município de Salvador, Bahia, Brasil. Hoehnea 18(2): 115 133.

NUNES, J. M. de C -1997- Hypneaceae, Rhodymeniaceae, Ceramiaceae e Rhodomelaceae (Rhodophyta) das praias de Placafor e Itapoã, Município de Salvador, Bahia, Brasil. Biotemas 10 (2): 61-75.

NUNES, J. M. de C -1998a- Rodofíceas marinhas bentônicas da orla oceânica de Salvador, Estado da Bahia, Brasil. Ínsula 27 (no prelo).

NUNES, J. M. de C -1998b- Catálogo de algas marinhas bentônicas do Estado da Bahia, Brasil. Acta Bot. Malacitana 23: 5-21.

NUNES, J. M. de C -1999- Phaeophyta da Região Metropolitana de Salvador, Bahia, Brasil. Dissertação de Mestrado, Universidade de São Paulo. 274p.

OLIVEIRA FILHO, E. C. de, Y. UGADIM \& E. J. de PAULA -1979- Comunidades associadas a plantas de Sargassum flutuantes em águas da corrente do Brasil - considerações biogeográficas. Boletim de Botânica da Universidade de São Paulo 7: 5-9.

TAYLOR, Wm. R -1930- Algae collected on the Hassler, Albatross and Schmidt Expeditions: I. Marine algae from Brazil. American Journal of 
Botany 16: 621-630.

TAYLOR, Wm. R -1931- A synopsis of the marine algae from Brazil. Revue Algologie 5: 279-313.

TEIXEIRA, V. L., R., C. PEREIRA, J. A. MUNIZ \& L. F. F. SILVA. da -1985- Contribuição ao estudo de algas de profundidade da costa sudeste do Brasil. Ciência e Cultura, 37(5): 809-815.

UGADIM, Y -1987- Distribuição das espécies de Gelidium e Pterocladia (Gelidiaceae Rhodophyta) no litoral brasileiro. Nerítica, 2(supl.): 65-74.

UGADIM, Y -1993- Taxonomia de Pterocladia (Gelidiaceae: Gelidiales: Rhodophyta) do Brasil. Revista Nordestina de Biologia 8(1): 527.

WYNNE, M. J -1998- A checklist of the benthic marine algae of the tropical and subtropical western Atlantic: first revision. Beiheft Nova Hedwigia 116: 1.155.
YAMAGUISHI-TOMITA, N -1970- Bolbocoleon jolyi, a new species of Chaetophoraceae (Chlorophyceae) from Redonda Island Abrolhos, eastern Brazil. Phycologia 9(2): 125-132.

Aceptado para su publicación en Octubre de 1999

Endereço do autor. Universidade Federal da Bahia Departamento de Botânica, Instituto de Biologia da, Campus de Ondina. CEP40.170-210, Salvador, Bahia, Brasil. E-mail: jmcnunes @ufba.br; Universidade do Estado da Bahia - Departamento de Ciências Exatas e da Terra - DCET, Campus II. Rodovia Alagoinhas/Salvador, km 03, CEP48.100000, Alagoinhas, Bahia, Brasil. 